\title{
Design and implementation of online electronic commerce questionnaire survey service platform \\ Wang Feng ${ }^{1, a}$,Ma Ning ${ }^{2, b}$ and Wan Fu cheng ${ }^{3,}$
}

Key Lab of China's National Linguistic Information Technology, Lanzhou,730030

a937859605@qq.com, b6105112@qq.com

Keywords: Echarts, MVC, Questionnaire survey

\begin{abstract}
Along with the development of science and technology, in the questionnaire service industry, began to gradually develop the Internet.More and more enterprises are inclined to make the decision of the company through online questionnaire survey service platform. The system uses JSP, MVC framework, MySQL database, Tomcat support service and other technologies to design an online questionnaire service platform. The user $\log$ in ,register and the management of the questionnaire, title, member information, announcement and message, as well as the realization of the results of the questionnaire will automatically generate Echarts has implemented.Through system developing and testing, this system basically achieves the goal of software design.Research shows that: use Echarts statistical survey results, more closely related with the data, the large amount of simplify artificial statistics, the same data type automatic summarize together, convenient for enterprises to carry out summary, analysis of the market, promote the development and prosperity of the enterprise, promote the process of survey service.
\end{abstract}

\section{Design background and significance}

IT tgechnology is more and more important, so that the enterprise also tend to the development of Internet gradually.More and more companies tend to through Internet count information on the company's decision. The company can through the Echarts generated statistical result analysis is helpful for company managers makeing decision to the company .The past practice was to organize a large number of people, to visit the streets, to inquire, to send questionnaires to the vast majority of people, through the results of statistical tables artificial analysis.But this kind of work, which requires a lot of manpower, material and time cost, is an unreasonable way of working for the company. With the rapid development of Internet, more and more companies are inclined to use IT technology to create a new working mode that can save money and material resources.

The questionnaire survey system provides management with a large number of compiledinformation statistics to facilitate the company's overall views and opinions on a certain area.Let the enterprise and the client have a better understanding of the project, specify the functional requirements of each part of the project, the data requirements, and clarify the implementation process of each function.

The performance of a web site is important for JSP programmers. The system has good performance and reduces the delay.For a large business type site, the performance is an important parameter of large sites can have housands of users browse per second, it requires good performance to satisfy users faster access, faster response speed, resulting in a good user experience.The online survey involves a wide range of people, with a large amount of data and a good performance to make the site more accessible to the public.

\section{Related technology introduction}

\subsection{MVC technology}

In 1979, Trygve Reenskaug first proposed the concept of MVC on the Smalltalk- 80 system, initially called model-view-controller-editor. 
In 1994, the Gang of Four made an in-depth analysis of the MVC pattern in the Design Patterns: Elements of Reusable object-oriented Software.

In MVC, $M$ is the model, which is used to process the business logic, to deal with the logic relationship between each module, and to do the actions of other modules when the state of a module changes. $\mathrm{V}$ is the view, which is used to handle the view layer, and when the logic layer changes, the vIEw is presented, so that the user can see the change visually.C is the controller, which is used to handle the control layer and connect the relationship between the logical layer and the view layer, using logic to call the corresponding view.

Let's take the login program as an example to introduce the workflow of MVC.Enter the login page and enter your account password.The login.jsp page submits the form to the actionservlet, the actionservlet reads the configuration file, finds the corresponding action, and finds the corresponding actionform through the pass value, which is submitted to the action based on the values that are passed. Action validates the value, invokes the corresponding method, and lets actionforward transfer the corresponding page.MVC provides the corresponding components for the Model, View, and Controller.

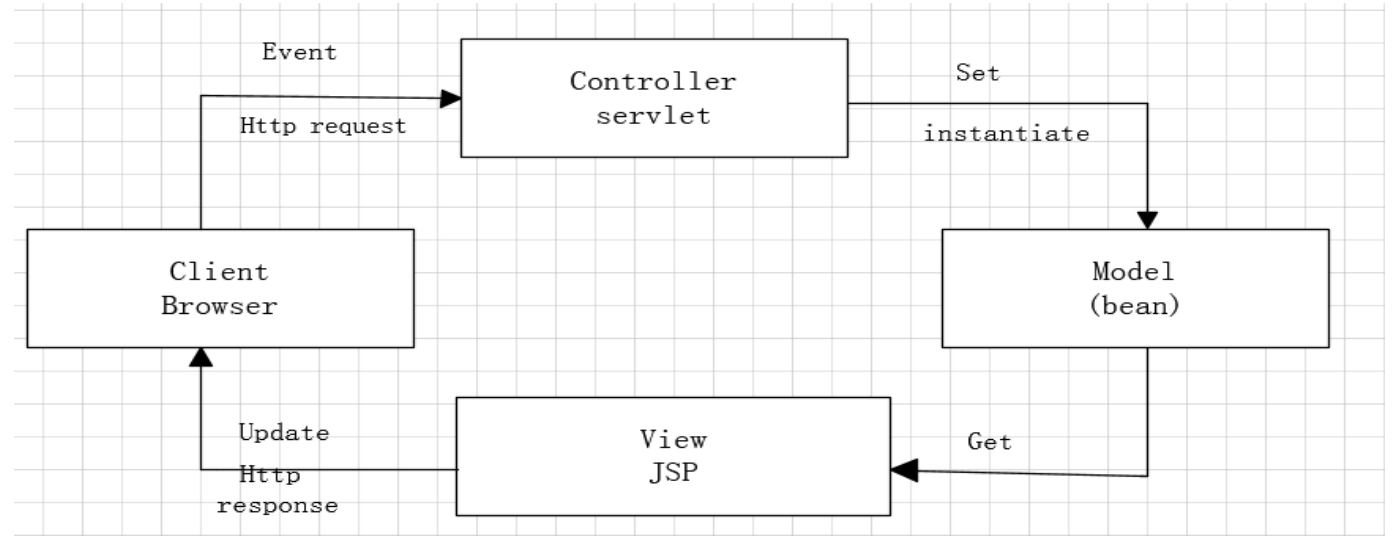

Figure2-1 Chart component diagram

\section{2 jQuery Technology}

jQuery is a lightweight code base in the Javascript base. It encapsulates a lot of in common method in the Javascript is very convenient to use.jQuery also contains CSS for development site staff is a good choice. Now many companies require employees to be familiar with jQuery technology.In addition, jQuery compatibility is also very good, which can be compatible with IE, openg, 360 security browser and safari as well provide ajax interaction. The biggest advantage of jQuery is that has a comprehensive API, which is very detailed for the use of various applications and plug-ins.This makes it is easy for developers to create beautiful pages.Currently, jQuery is very popular around the world, and most of the website construction is used in jQuery technology.It is conceivable the importance of jQuery technology.

Created by the American John Resig, jQuery use a number of commonly used methods in the original Javascript to encapsulate it and make it become a handy tool for everyone. We don't need a detailed understanding of the internal specific code, which saves time for development.

JQuery contains a large number of plug-ins to support a variety of fine effect which allows the programmer to make cool animation static elegant web pages easily.In writing the code reference jQuery that don't need to know the specific code writing. The programmer reduces the operating difficulty, but also accelerates the speed of code written.

Currently, jQuery has two versions, one is uncompressed and the other is mini.The uncompressed version is more formal and comprehensive.It contains a lot of Javascript code, which is applied to the development to achieve the desired effect, while the mini version is used after the development.The mini-pane contains less Javascript code, faster downloads, fewer files to load than uncompressed versions, and faster operation. 
The advantages of jQuery are obvious, which has led to It's rapid popularity, and there are more and more programmers using the jQuery technology develop website.The purpose of jquery is to do more thing with less code.

The jQuery UI is the code that abstracts from the interaction of a page in a jQuery Javascript library.It's a toolbox for page interaction.If you want to build a page with a high level of interaction. It's easy to use jQuery UI, which is a good choice for developers who work on UI dispose .It contains basic jQuery that enables developers to easily handle tags, practices, and provide ajax interactions.All plug-in tests are compatible with IE 6.0+, Firefox 3+, Safari 3.1+, Opera 9.6+, and Google Chrome.

\subsection{Echarts}

Echarts abbreviated from Enterprise Charts(business level data chart) which is a pure Javascript libraries. Echarts's API Introduction is fully intact which is in collaboration with MyEclipse unobstructed only need code reference js. The display is also very smooth in the browser.Echarts are compatible with multiple browsers such as IE, Google, firefox, 360 security, and safari currently.Echarts can drag for calculating sum and average. Echarts view support marca dragon theme, line is fluent and support for multiple components.Echarts website also has a lot of examples for reference and modify applications which is very practical as well bring convenient to the user's experience.
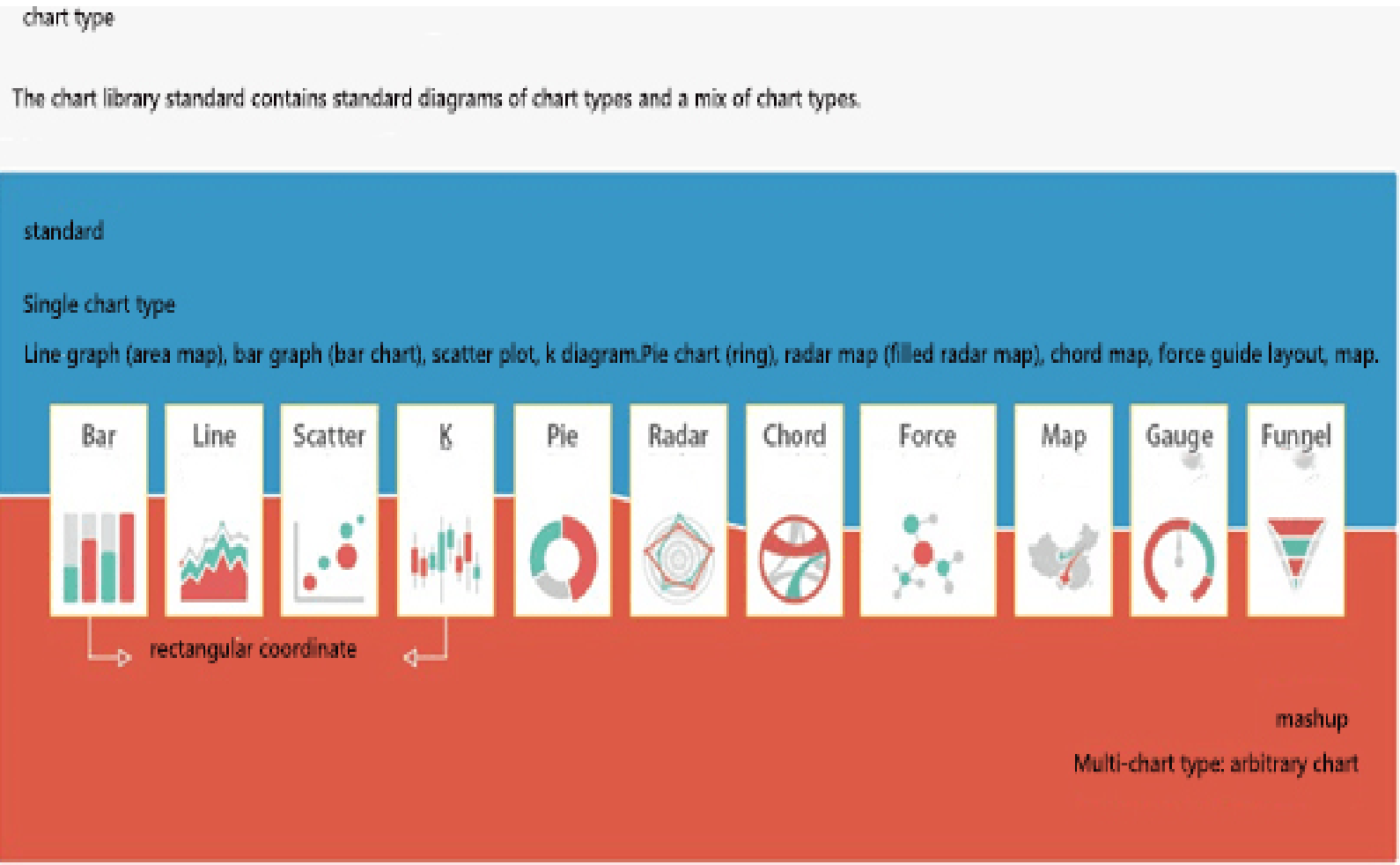


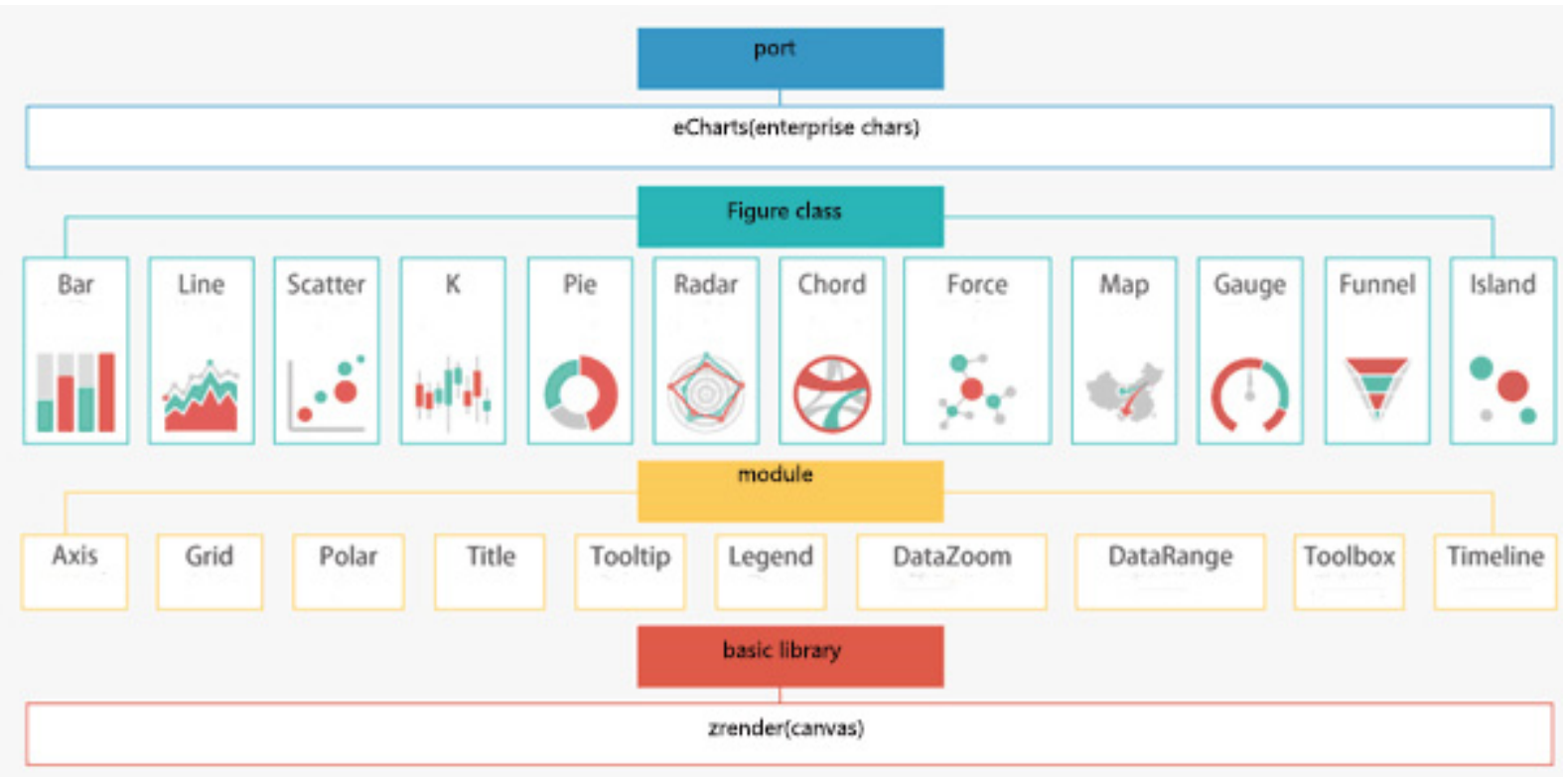

Figure 2-2 diagram component diagram

\section{Design concept and scheme}

\subsection{Overview of system functions}

The questionnaire survey management system is for the company to analyze the statistical results generated by Echarts, which is beneficial to the judgment of the company's managers on the decision-making of the company. Past practice is to organize a large number of staff, visited on the street in order to send the general survey statistics to people.By the result of statistics can help for the company decision-making function mainly includes the following aspects:

Ordinary users module: users can browse the questionnaire, answer the questionnaire, announcement,look over.

Release modules: user login, delete the questionnaire, the registration information change, and delete message board.

System administrator module: password can be modified, registered users delete, questionnaire of edit, delete, classification of questionnaire operation, delete release people, edit, delete announcement and edit, and delete message board.

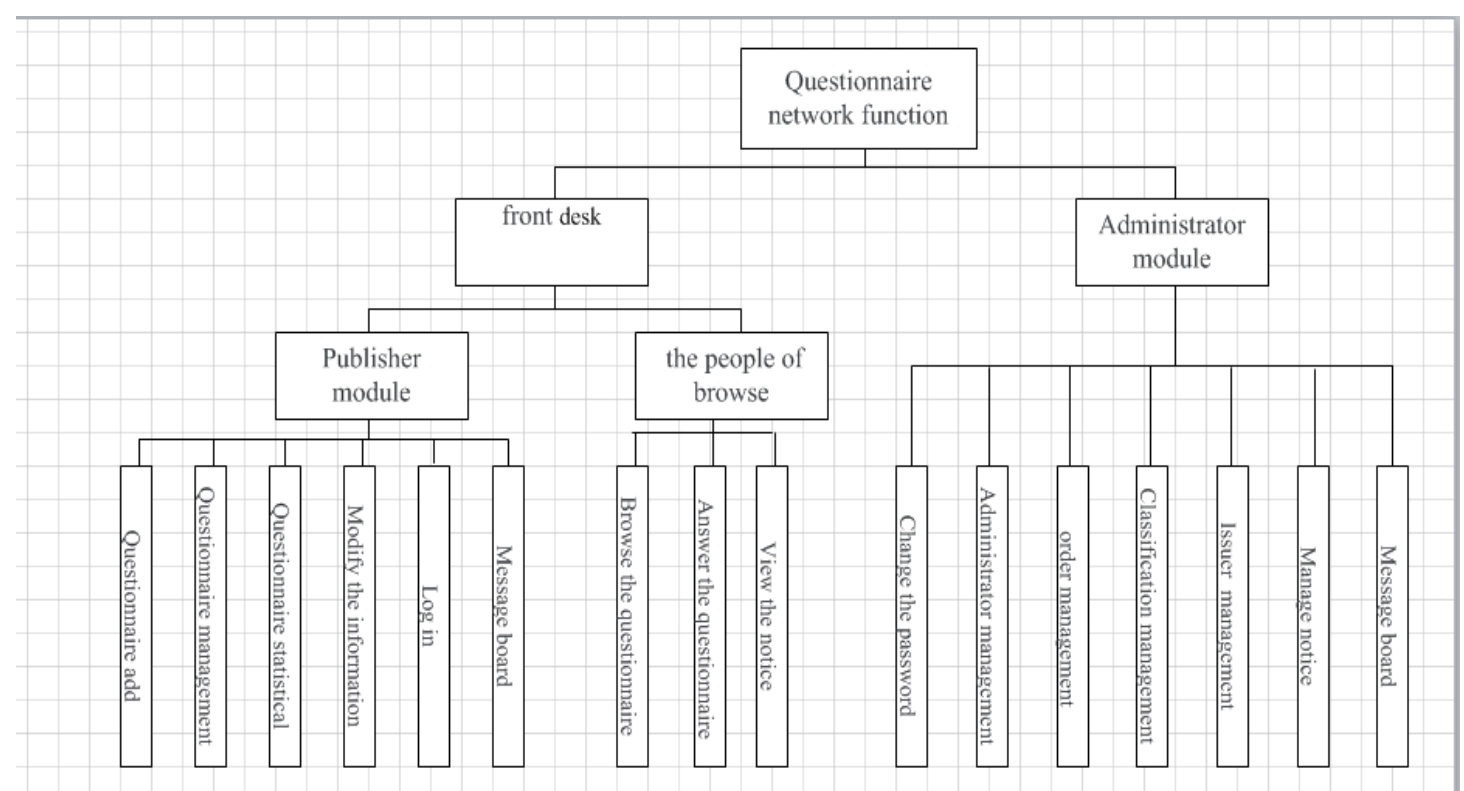

Figure 3-1 system function module diagram 


\subsection{Development platform construction}

Build the development environment: mainly rely on MyEclipse for development.At the first to install the JDK, and then to deal the JDK configuration environment variables.

This web site needs a backend server.So you need to download and install Tomcat server, and then start Tomcat. Then download and install MySQL, set the user's name and password of the database, and then start MySQL.

Download and install MyEclipse, at the same time MyEclipse binding JDK, Tomcat and MySQL.

\subsection{Core function module}

The user enters the website to display the homepage of the website, can register, log on the website in order to becoming the publisher.Publisher can conduct questionnaire management, preview, delete, and edit.

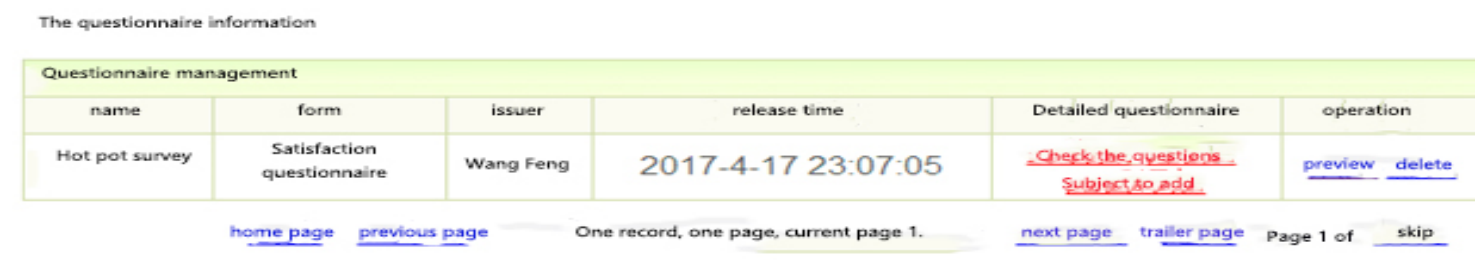

Figure 3-2 questionnaire management chart

Questionnaire management code:

mydb.doPstm(sql, params);

ResultSet $r s=$ mydb.getRs () ;

for (int $\mathrm{i}=0 ; \mathrm{i}<$ (page -1$) *$ EVERYPAGENUM; $\mathrm{i}++)\{$

rs.next();

\}

for (int $\mathrm{t}=0 ; \mathrm{t}<$ EVERYPAGENUM; $\mathrm{t}++$ ) \{

if $($ rs.next ()$)\{$

Twenjuan wenjuan=new Twenjuan();

wenjuan.setId(rs.getString("id"));

wenjuan.setTitle(rs.getString("title"));

wenjuan.setType_id(rs.getInt("type"));

wenjuan.setType_name(liuService.getTypeName(rs.getInt("type")));

wenjuan.setContent(rs.getString("content"));

wenjuan.setShijian(rs.getString("shijian"));

wenjuan.setUser_id(rs.getInt("userid"));

wenjuan.setUser_name(liuService.getPutuserName(rs.getInt("userid")));

wenjuanList.add(wenjuan);

\} else \{

break;

\}

\}

rs.close ();

The viewer refers to a user who has no registration. The user has no special permission and can only browse the questionnaire and fill in the questionnaire.

Questionnaire is editted by publisher.Answer the questions above, and then submit.

This user is used to provide information to the publisher and the background administrator for Echarts summarizes the data and gives a general understanding of the public perception. 


\section{Hot pot survey \\ Satisfaction questionnaire $\quad 2017-4-17$ 23:07:05}

1. Multiple Choice

What do you like to eat?
A: $\bigcirc$ hot pot
B: seafood
C. $\mathrm{S}$ snack
D: $\bigcirc$ home cookin

2. Multiple Choice

How do you like the self-service
A: $\bigcirc$ good
$B: O$ ordinary
C. $\bigcirc$ A little bad
D: $\bigcirc$ disgusting

3. Multiple Choice

You eat hotpot's faworite hotness.
A: $\bigcirc$ Abnormal hot
B: $\bigcirc$ hot

Figure3-3 questionnaire

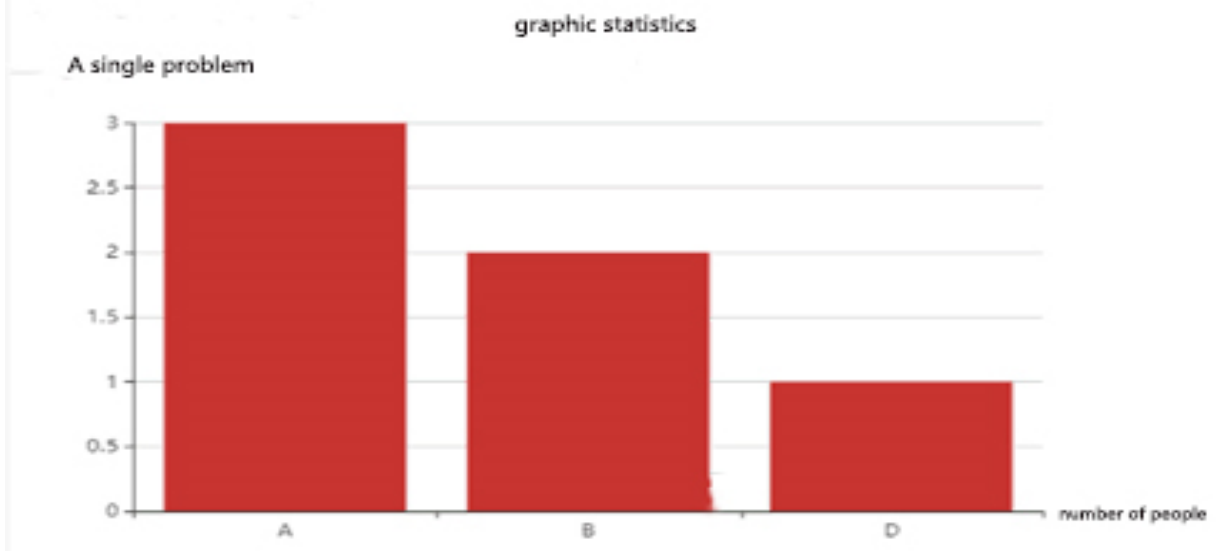

A multiple-choice question

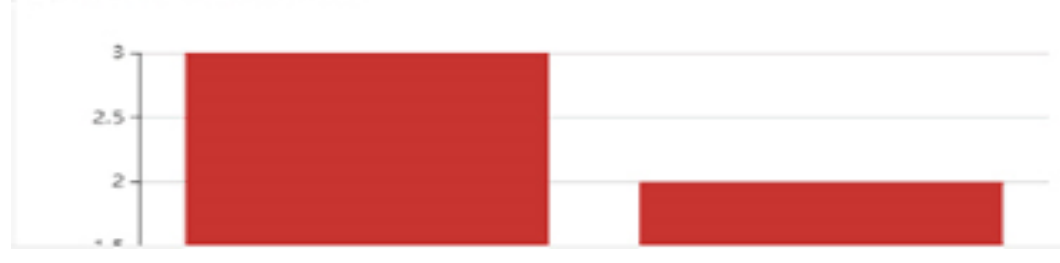

Figure 3-4 chart of Echarts

Questionnaire completion code:

String id=String.valueOf(new Date().getTime());

String title=req.getParameter("title");

String type_id=req.getParameter("fenlei_id");

String content=req.getParameter("content");

String userid=req.getParameter("userid");

String shijian=req.getParameter("shijian"); 
String gqdate=req.getParameter("gqdate");

String del="no";

String sql="insert into t_wenjuan values(?,?,?,?,?,?,?,?)";

Object[] params $=\{$ null, title,shijian,type_id,content,del,userid,gqdate $\}$;

DB mydb=new DB () ;

mydb.doPstm(sql, params);

mydb.closed();

The background administrator can change the information such as administrator password through login, and conduct questionnaire management as well classify the questionnaire.

\begin{tabular}{|c|c|}
\hline \multicolumn{1}{|c|}{ name } & operation \\
\hline Satisfaction questionnaire & delete \\
\hline Questionnaire type 2 & deate \\
\hline Test categorization & dente \\
\hline
\end{tabular}

\section{additio}

Questionnaire classitication code:

String type=req.getParameter("type");

if(type.endsWith("fenleiMana"))

\{

fenleiMana(req, res);

\}

if(type.endsWith("fenleiAdd"))

\{

fenleiAdd(req, res);

\}

if(type.endsWith("fenleiDel"))

\{

fenleiDel(req, res);

\}

\section{Conclusion}

This system uses Eclipse as the development software,Tomcat, MySQL as support, realized the basic function of online questionnaire survey.It is proved by practice that Echarts can be used in the questionnaire survey service to make the questionnaire results easier to collate and summarize. The related types of data are summarized together, the background conduct calculating and the collection of all aspects is obtained, the Echarts graph is formed at the same time.The function of absolute data collection and data summarization is more intuitive and convenient to reflect the market rules and the interests of some small groups.Has the meets the market, the public demand and the practical value.At present, there are many shortcomings and vulnerabilities in the system. The page of the website needs to be further improved.I need to make further adjustments to my code. The function of each role of the system is duplicated, and the role's function needs to be further divided.

\section{Acknowledgement}

This research is supported by National Science-technology Support Plan Projects(NO. 2015BAD29B01) 


\section{References}

[1] Tang Xiaodan and Tang Ziying, Computer Operating System [M],Xi 'an: xi 'an electronic science and technology university press, pp.26-45, 2007.

[2] Wang Yingqiang, Chen Suiyang,and Zhang Wensheng,Android programming [M],Beijing: tsinghua university press, pp.18-253, 2013.

[3] Qian Xuanzhong and Li Jing,Principle and application of database [M],Beijing: Beijing university of posts and telecommunications press,pp.178-210,2010.

[4] Chen Tianhe,Struts Hibernate Spring integrated development [M],Electronic industry press,pp.7-18, 2007.

[5] Sun Weiqin,Java object-oriented programming [M],Beijing: electronic industry press,pp.121-239,2006.

[6] Marty Hall,Serlet and JSP core programming [M], Mechanical industry press,pp.2-3,2008.

[7] Bruce Eckel,Thingking in Java[M],America:Prentice Hall PTR,pp.134-233,2006.

[8] Reinnberg,JunIT Recipes Chinese: programmer practical test techniques [M], Beijing: electronic industry press,pp.9-24,2010.

[9] $\mathrm{Wu}$ Bird, Exploration and research on the practical training platform of the Web application development course for sharing school-enterprise cooperation [J],Technology wind,2015(01).

[10]Lin Feng,Echarts introduction to mastery [M], Beijing: people's post and telecommunications press, pp.11-21,2009.

[11]Li Chengyuan,A series of jQuery front-end development series [M], People's post and telecommunications press,pp.56-71,2010.

[12] Wang Yunfei,The subversive Spring Boot practice of JavaEE development [M], Electronic industry publishing house,pp.89-97,2012. 